\title{
Inhibitory Activity on Amyloid Aggregation of Rosmarinic Acid and Its Substructures From Isodon japonicus
}

Natural Product Communications May 2019: 1-5 (c) The Author(s) 2019

Article reuse guidelines: sagepub.com/journals-permissions DOI: $10.1177 / 1934578 \times 19843039$ journals.sagepub.com/home/npx

\author{
Jiayi Sun', Gandan Jiang', and Hideyuki Shigemori ${ }^{2}$
}

\begin{abstract}
Nowadays, the incidence of Alzheimer's disease (AD) and type 2 diabetes (T2D) is increasing at an alarming rate. More and more studies have been investigating the relationship between these two diseases and are trying to find an effective treatment. According to amyloid hypothesis, it is very necessary to find phenolic compounds with catechol moieties, which would inhibit the aggregation of amyloid $\beta$ (A $\beta$ ) and human islet amyloid polypeptide (hIAPP), while also exhibiting antioxidant activity and protective effect. We isolated rosmarinic acid (RA) from the plant Isodon japonicus (Burm.f.) H. Hara. Thioflavin T assay and transmission electron microscopy observation were carried out to evaluate the inhibitory effect of RA, caffeic acid, and 3,4-dihydroxyphenyllactic acid, which are the substructures of RA, on both A $\beta$ and hIAPP fibrillization. 2,2-Diphenyl-Ipicrylhydrazyl assay was applied to test the antioxidant activity. RA showed inhibitory effect on both peptides and strong antioxidant activity. These results suggest that the existence of catechol units plays an important role on the inhibitory activity. Therefore, RA will be a promising strategy to prevent AD and T2D.
\end{abstract}

\section{Keywords}

Alzheimer's disease, type 2 diabetes, amyloid $\beta$, human islet amyloid polypeptide, rosmarinic acid

Received: September 3rd, 2018; Accepted: December 13th, 2018.

Alzheimer's disease (AD) and diabetes are parallel chronic diseases arising from aging, and the incidence is at an alarming rate, thereby becoming a highly concerning global health issue. Insulin resistance is considered as a core feature of type 2 diabetes (T2D), and emerging as a potentially important feature of AD. ${ }^{1}$ Some environmental factors and susceptibility genes lead to insulin resistance, which lets diabetes uncontrolled. Too much sugar remaining in the blood may also damage the vessels and organs including the brain, furtherly cause $\mathrm{AD}{ }^{2}{ }^{\mathrm{T}} \mathrm{T} 2 \mathrm{D}$ and $\mathrm{AD}$ can both lead to cognitive decline, as well as brain hypofunction, memory disturbance, blindness, and so on.

Although the exact pathological defects in $\mathrm{AD}$ and $\mathrm{T} 2 \mathrm{D}$ remain unknown, the prevailing theories implicate that the aggregation of amyloid $\beta(A \beta)$ and human islet amyloid polypeptide (hIAPP) leads to such diseases. A $\beta$ is a peptide containing 36 to 43 amino acids, which are formed from cleavage of amyloid precursor protein. hIAPP is a 37-residue regulatory peptide, also the major secretory product of $\beta$-cells. Both peptides show a similar aggregation principle, and the aggregation of $A \beta$ causes the extreme shrinkage of cerebral cortex and hippocampus in the brain, while hIAPP aggregation leads to the $\beta$-cell destruction.
Isodon japonicus (Burm.f.) H. Hara (Labiatae) is often applied in traditional Chinese and Japanese medicine, and it mainly grows in Northeast Asia. The aerial part of the plant is often used for the treatment of gastrointestinal disorders, ${ }^{3}$ while its dried leaves show strong antioxidant activity, ${ }^{4}$ antiallergic activity, ${ }^{5}$ tanning activity, ${ }^{6}$ and melanogenic inhibitory activity. ${ }^{7}$ Besides diterpenoids, I. japonicus is also a rich source of phenolic compounds. Rosmarinic acid (RA) is one of the dominant compounds in the plant, which is the dimer of caffeic acid (CA) and with two catechol moieties.

In our previous studies, we reported that 4,5-di- $O$-caffeoylquinic acid and 3,4,5-tri- $O$-caffeoylquinic acid strongly inhibited the aggregation of $\mathrm{A} \beta 42$ in a dose-dependent manner. ${ }^{8}$ Acteoside and oraposide were also found

\footnotetext{
' Graduate School of Life and Environmental Sciences, University of Tsukuba, Ibaraki, Japan

${ }^{2}$ Faculty of Life and Environmental Sciences, University of Tsukuba, Ibaraki, Japan

Corresponding Author:

Hideyuki Shigemori, Faculty of Life and Environmental Sciences, University of Tsukuba, Ibaraki 305-8572, Japan.

Email: shigemori.hideyuk.fn@u.tsukuba.ac.jp
} 


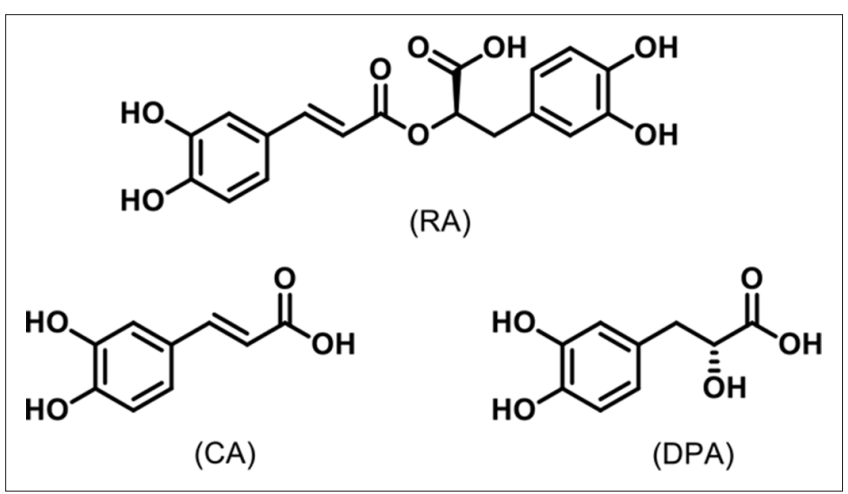

Figure I. Chemical structures of RA, CA, and DPA. CA, caffeic acid; DPA, 3,4-dihydroxyphenyllactic acid; RA, rosmarinic acid. CA, caffeic acid; DPA, 3,4-dihydroxyphenyllactic acid; RA, rosmarinic acid.

to exhibit strong activities for inhibition of $A \beta$ aggregation and antioxidant activity. ${ }^{9,10}$ Some phenolic compounds with catechol moieties were proved possessing the inhibitory activity on the aggregation of $\mathrm{A} \beta$. Recently, we found that glucuronosylated flavonoids with a catechol moiety exhibited potent inhibitory activity of $\mathrm{A} \beta$ and hIAPP aggregations. ${ }^{11}$ On the other hand, RA shows strong antioxidant activity, and it can be expected to possess more biological activities also. In this study, we aimed on investigating the protective effect of RA, CA, and 3,4-dihydroxyphenyllactic acid (DPA) (Figure 1), which are the substructures of RA, on both AD and T2D. We successfully found that RA displayed inhibitory activity on amyloid aggregation related to both diseases.

To determine the inhibitory activity of RA, CA, and DPA against $A \beta$ and hIAPP fibrillizations, Thioflavin $T$ (Th-T) assay was used to measure the efficiency of these compounds. Among all the samples dealt with different concentration, these compounds expressed the concentration-dependent effect on protein fibrillization. As shown in Figure 2(a)-(c), RA showed the most significant

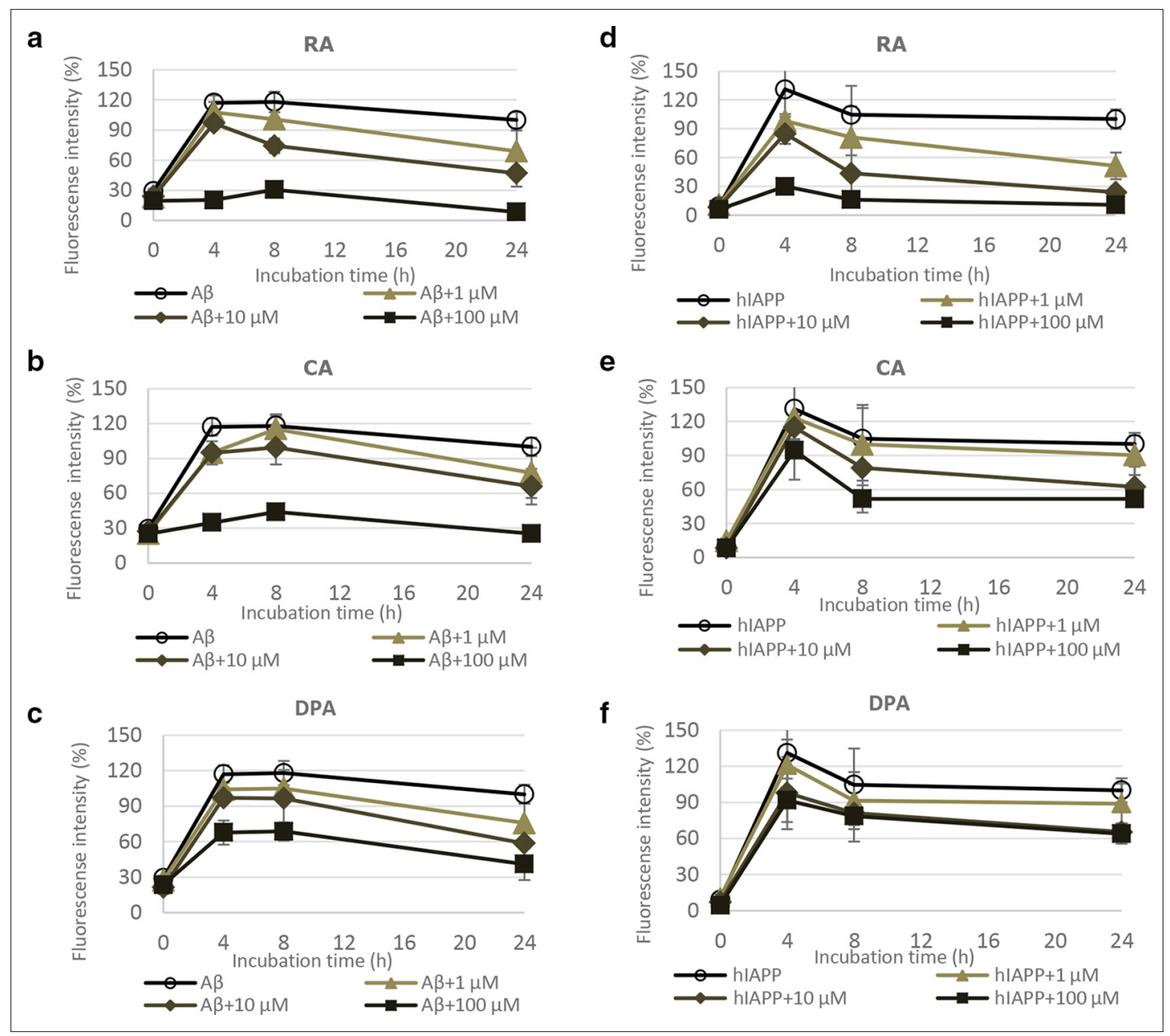

Figure 2. Effect of RA, CA, and DPA on the Th-T fluorescence from $A \beta$ and hIAPP. (a) A $\beta 42$ with RA; (b) $A \beta 42$ with CA; (c) A $\beta 42$ with DPA; (d) hIAPP with RA; (e) hIAPP with CA; (f) hIAPP with DPA. A $\beta$, amyloid $\beta$; CA, caffeic acid; DPA, 3,4-dihydroxyphenyllactic acid; hIAPP, human islet amyloid polypeptide; RA, rosmarinic acid; Th-T,Thioflavin T. 


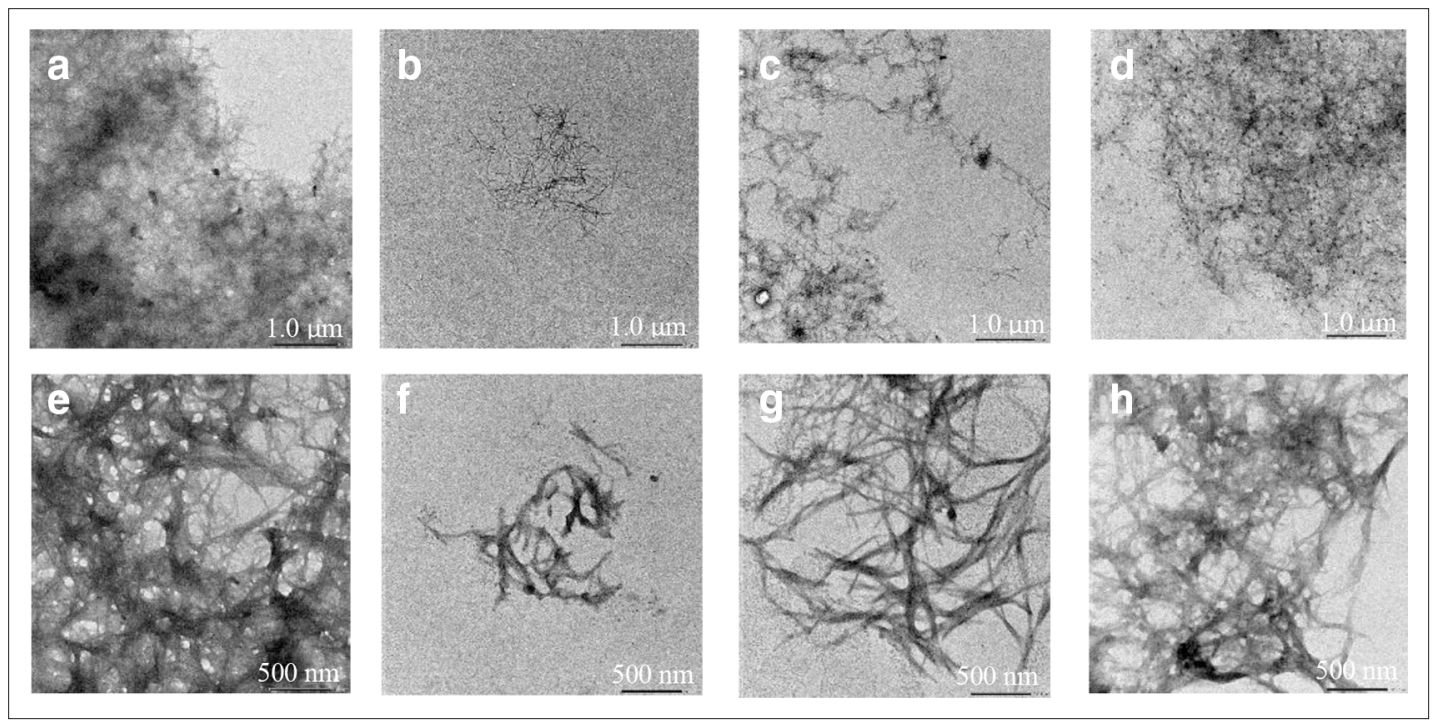

Figure 3. TEM observation of A $\beta$ and hIAPP. (a) $25 \mu \mathrm{MA} \beta 42$; (b) $25 \mu \mathrm{MA} \beta 42$ with $100 \mu \mathrm{M} \mathrm{RA}$; (c) $25 \mu \mathrm{MA} \beta 42$ with I00 $\mu$ M CA; (d) $25 \mu \mathrm{MAB} 42$ with $100 \mu \mathrm{M}$ DPA; (e) $25 \mu \mathrm{M}$ hIAPP; (f) $25 \mu \mathrm{M}$ hIAPP with $100 \mu \mathrm{M}$ RA; (g) $25 \mu \mathrm{M}$ hIAPP with I00 $\mu \mathrm{M}$ CA; (h) $25 \mu \mathrm{M}$ hIAPP with $100 \mu$ M DPA. A $\beta$, amyloid $\beta$; CA, caffeic acid; DPA, 3,4-dihydroxyphenyllactic acid; hIAPP, human islet amyloid polypeptide; RA, rosmarinic acid;TEM, transmission electron microscopy.

inhibitory activity on $\mathrm{A} \beta 42$ aggregation, whose $\mathrm{IC}_{50}$ value was $4.8 \mu \mathrm{M}$, while CA exhibited a stronger inhibitory activity $\left(\mathrm{IC}_{50} 17.9 \mu \mathrm{M}\right)$ than that of DPA on $\mathrm{A} \beta 42\left(\mathrm{IC}_{50} 31.2 \mu \mathrm{M}\right)$. $\mathrm{CA}$ is also used as a positive control in our previous reports. ${ }^{8,10}$ Figure 2(d)-(f) depicts the inhibitory activity on hIAPP aggregation of RA, CA, and DPA. RA and CA showed the inhibitory effect on hIAPP fibrillization at $\mathrm{IC}_{50} 3.1$ and $57.6 \mu \mathrm{M}$, respectively. The effect of DPA was not significantly detected $\left(\mathrm{IC}_{50}>100 \mu \mathrm{M}\right)$. But different from $\mathrm{A} \beta$, the formation of hIAPP fibril mostly happened in the first 4-hour incubation, after reaching the maximum of Th- $\mathrm{T}$ fluorescence intensity at 4 hours, RA, CA, and DPA started exhibiting the effect on the fibril formation of hIAPP relatively, and after 8 hours the hIAPP entered the stable phase.

Transmission electron microscopy (TEM) images indicated the typical fibrillization of $A \beta$ and hIAPP incubated with or without RA, CA, and DPA. Proteins incubated without these compounds showed more intensive fibril formation than those incubated with the compounds. Figure 3(b), (f) shows the ability of RA on inhibiting the fibril formation of A $\beta 42$ and hIAPP which was the most significant with a sparse fibrillization image. CA (Figure 3(c) and (g)) and DPA (Figure $3(\mathrm{~d})$ and $(\mathrm{h})$ ) also affect the aggregation of both protein fibrils, but not that strong as RA.

Polyphenols affect the aggregation of $A \beta$ and hIAPP due to the block of the aromatic ring on $\pi-\pi$ stacking $^{12}$ and the connection of quinone (auto-oxidized from catechol unit) and amyloid monomer, due to Michael addition or the imine formation. ${ }^{13}$ In the structure of RA, two catechol moieties are involved, which indicate that more blocks and connection exist between RA and protein chains. Comparing DPA with CA containing a carbon double bond means that is more easily auto-oxidized, possessing a stronger ability on connecting with amyloid monomers.

Besides inhibiting the aggregation of $\mathrm{A} \beta$ and hIAPP, the compound with antioxidant activity can also prevent the cell destruction related to AD and T2D. Reactive oxygen species (ROS) is always produced by living organisms because of the cellular metabolism and environmental factors. ${ }^{14}$ In the AD and T2D, ROS can lead to the death of neuronal cells in the brain and also to $\beta$-cell destruction, which is related to insulin production. Oxidative stress, the shift of the balance between oxidants and antioxidants, contributes to many diseases including neurological disorders, diabetes, and so on.

2,2-Diphenyl-1-picrylhydrazyl (DPPH) radical scavenging assay was carried out to evaluate the antioxidant activity of RA, CA, and DPA, and all these compounds express the concentration-dependent antioxidant activity. As shown in Figure 4, RA shows the strongest antioxidant activity, the antioxidant rate reaching more than $80 \%$ with concentrations of 25 and $50 \mu \mathrm{M}$, even more significant than the positive control. DPA also shows the strong antioxidant activity $(65 \%$ at $50 \mu \mathrm{M})$, while the antioxidant activity of CA is not significant $(25 \%$ at $50 \mu \mathrm{M})$.

Bendary et al. found that the antioxidant activity is related to the compound structure, which in turn depends on the number and position of the functional groups such as hydroxyl group, amino group, and so on. ${ }^{15}$ In the structures of RA, CA, and DPA, RA has the most OH groups, and DPA includes more hydroxyl groups than $\mathrm{CA}$. This may be the reason that the antioxidant intensity is ranked in RA, DPA, and CA.

In the present study, some relationship between $\mathrm{AD}$ and T2D has been found. Because $\mathrm{A} \beta$ and hIAPP play an 


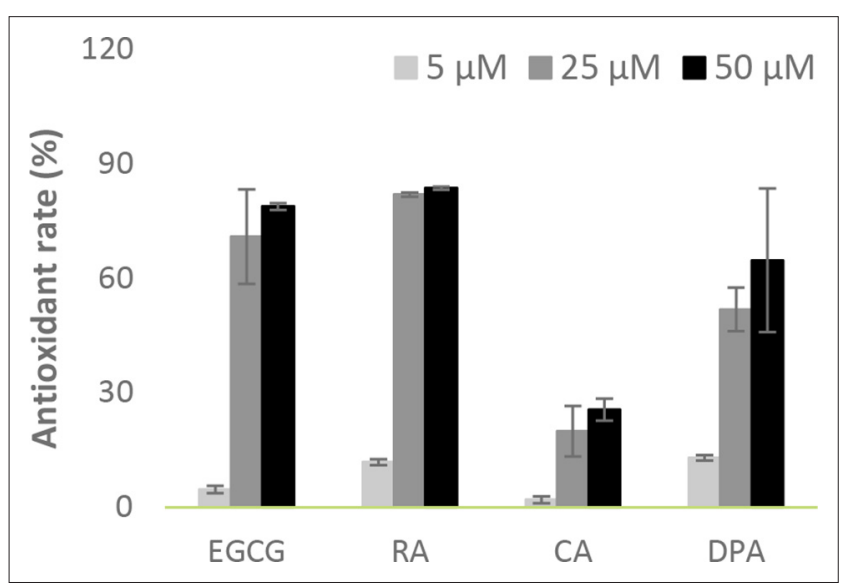

Figure 4. Antioxidant activity of RA, CA, and DPA. CA, caffeic acid; DPA, 3,4-dihydroxyphenyllactic acid; EGCG, epigallocatechin gallate; RA, rosmarinic acid.

important role in triggering $\mathrm{AD}$ and $\mathrm{T} 2 \mathrm{D}$, we suggested that it be necessary to find the compounds that can inhibit the protein fibrillization and show a strong antioxidant activity. In the structure of RA, CA, and DPA, the catechol moieties showed a significant influence on the inhibition of amyloid aggregation, while the hydroxyl groups expressed strong antioxidant activities. Both inhibitory activity and antioxidant activity may show the protective effect and lead to efficient therapy related on $\mathrm{AD}$ and $\mathrm{T} 2 \mathrm{D}$.

Also, as per the several reports on RA about its inhibitory activity toward $A \beta$ and hIAPP aggregation, ${ }^{16,17}$ we believed that applying RA in medicine will be a promising strategy to treat and prevent $\mathrm{AD}$ and diabetes. The findings have inspired us to conduct more investigation on various biological activities of phenolic compounds from other medicinal plants apart from I. japonicus to get more useful guidelines in relation to search for novel RA-related inhibitors against protein aggregation associated with chronic diseases.

\section{Experimental}

\section{Isolation of RA}

Isodon japonicus (Burm.f.) H. Hara was purchased from Kanai Co., Ltd. (Tokyo, Japan). After smashing, the dried I. japonicus $(100 \mathrm{~g})$ was extracted with $\mathrm{MeOH}(700 \mathrm{~mL} \times 2)$ at room temperature for 24 hours. After filtration and evaporation, the $\mathrm{MeOH}$ extract was mixed with EtOAc $(400 \mathrm{~mL} \times 3)$ and $\mathrm{H}_{2} \mathrm{O}$ $(400 \mathrm{~mL})$. A portion $(2 \mathrm{~g})$ of EtOAc-soluble material (RJ-EA, $4.37 \mathrm{~g}$ ) was chromatographed over octadecylsilyl (ODS) column (Cosmosil 75 $\mathrm{C}_{18}$-PREP, $\phi 2.2 \times 30 \mathrm{~cm}$, acetone: $\mathrm{H}_{2} \mathrm{O}=$ $3: 7 \rightarrow 1: 0)$. RJ-EA-4 $(260.5 \mathrm{mg})$ was separated using ODS column $\left(\right.$ Cosmosil $75 \mathrm{C}_{18}$-PREP, $\phi 1.0 \times 30 \mathrm{~cm}, \mathrm{MeOH}: \mathrm{H}_{2} \mathrm{O}=$ $3: 7 \rightarrow 1: 0)$ to afford RJ-EA-4-1 4-8. RJ-EA-4-3 (23.5 mg) was purified by TSKgel ODS- $120 \mathrm{~A}$, MeOH- $1 \% \mathrm{AcOH}$ in water (30:70) to obtain RA (11.1 mg) and confirmed by ${ }^{1} \mathrm{H}$ NMR spectrum $\left(\mathrm{CD}_{3} \mathrm{OD}, 500 \mathrm{MHz}\right)$.

CA and DPA were purchased from FUJIFILM Wako Pure Chemical Corporation, Japan.

\section{Th-T Fluorescence Assay}

$\mathrm{A} \beta 42$ was dissolved in $0.1 \% \mathrm{NH}_{4} \mathrm{OH}$ and hIAPP (KareBay Biochem Inc., USA) was dissolved in 1,1,1,3,3,3-hexafluoro-2-propanol. The amyloid solution was diluted 10-fold with $50 \mathrm{mM}$ phosphate-buffered saline (pH 7.4). Both peptides were pretreated with 1,10 , and $100 \mu \mathrm{M}$ of RA, CA, and DPA, in the 96-well microtiter plate (Thermo Scientific), respectively. All the samples were incubated for 24 hours at $37^{\circ} \mathrm{C}$. Th-T fluorescence intensity was evaluated at $0,4,8$, and 24 hours, and found to show an excitation wavelength of $420 \mathrm{~nm}$ and an emission wavelength of $485 \mathrm{~nm}$.

\section{Transmission Electronic Microscopy}

A 342 and hIAPP (each $25 \mu \mathrm{M}$ ) treated with equimolar RA, $\mathrm{CA}$, and DPA were spotted onto a carbon-coated Formvar grid and incubated for 2 minutes at room temperature and air-dried for 5 minutes after washing by $\mathrm{H}_{2} \mathrm{O}$ twice and $0.4 \%$ silicotungstic solution twice. Samples were observed under the JEOL JEM-1400 electron microscope.

\section{DPPH Radical Scavenging Assay}

DPPH radical scavenging assay was applied to evaluate the antioxidant activity of RA, CA, and DPA. The positive control epigallocatechin gallate and the three compounds were pretreated to 5,25 , and $50 \mu \mathrm{M}$, and $10 \mu \mathrm{L}$ of each sample was injected into a 96-well plate. Half were treated with 190 $\mu \mathrm{L}$ of DPPH solution [combined DPPH $(1.9 \mathrm{mg}) / 12.0 \mathrm{~mL}$ EtOH, $3.0 \mathrm{~mL}$ of 2-( $N$-morpholino)ethanesulfonic acid (MES), and $9.0 \mathrm{~mL}$ of $\mathrm{H}_{2} \mathrm{O}$ ], and half were treated without DPPH (combined EtOH $1.0 \mathrm{~mL}, 3.0 \mathrm{~mL}$ of MES, and $9.0 \mathrm{~mL}$ of $\mathrm{H}_{2} \mathrm{O}$ ). After a $15 \mathrm{~min}$ reaction at room temperature, both samples were examined under the wavelength of $490 \mathrm{~nm}$.

\section{Acknowledgments}

We thank Professor Kazuhiro Irie, Associate Professor Kazuma Murakami, and Dr Mizuho Hanaki, Graduate School of Agriculture, Kyoto University for preparing A $\beta 42$.

\section{Declaration of Conflicting Interests}

The author(s) declared no potential conflicts of interest with respect to the research, authorship, and/or publication of this article.

\section{Funding}

The author(s) disclosed receipt of the following financial support for the research, authorship, and/or publication of this article: this 
work was partially supported by JSPS KAKENHI Grant Number JP24580156.

\section{References}

1. Arnold SE, Arvanitakis Z, Macauley-Rambach SL, et al. Brain insulin resistance in type 2 diabetes and Alzheimer disease: concepts and conundrums. Nat Rev Neurol. 2018;14(3):168-181.

2. Gray SM, Meijer RI, Barrett EJ. Insulin regulates brain function, but how does it get there? Diabetes. 2014;63(12):3992-3997.

3. Fujita E, Node M, Johne S, Kasai R, Tanaka O. Diterpenoids of Rabdosia species. Fortschr Chem Org Naturst. 1984;46:77-157.

4. Masuoka N, Isobe T, Kubo I. Antioxidants from Rabdosia japonica. Phytother Res. 2006;20(3):206-213.

5. Inyushkina YV, Bulgakov VP, Veselova MV, et al. High rabdosiin and rosmarinic acid production in Eritrichium sericeum callus cultures and the effect of the calli on masugi-nephritis in rats. Biosci Biotechnol Biochem. 2007;71(5):1286-1293.

6. Agata I, Hatano T, Nishibe S, Okuda T. A tetrameric derivative of caffeic acid from Rabdosia japonica. Phytochemistry. 1989;28(9):2447-2450.

7. Nitoda T, Isobe T, Kubo I. Effects of phenolic compounds isolated from Rabdosia japonica on B16-F10 melanoma cells. Phytother Res. 2008;22(7):867-872.

8. Miyamae Y, Kurisu M, Murakami K, et al. Protective effects of caffeoylquinic acids on the aggregation and neurotoxicity of the 42-residue amyloid $\beta$-protein. Bioorg Med Chem. 2012;20(19):5844-5849.
9. Kurisu M, Miyamae Y, Murakami K, et al. Inhibition of amyloid $\beta$ aggregation by acteoside, a phenylethanoid glycoside. Biosci Biotechnol Biochem. 2013;77(6):1329-1332.

10. Shigemori H, Kidachi E, Kurisu M, et al. Structure-activity relationship of phenylethanoid glycosides on the inhibition of amyloid $\beta$ aggregation. Heterocycles. 2016;92(11):1976-1982.

11. Ben Hmidene A, Hanaki M, Murakami K, Irie K, Isoda H, Shigemori H. Inhibitory activities of antioxidant flavonoids from Tamarix gallica on amyloid aggregation related to Alzheimer's and type 2 diabetes diseases. Biol Pharm Bull. 2017;40(2):238-241.

12. Matsuda Y, Irie K. Polyphenols as potential preventive agents for Alzheimer's disease.. Foods \& Food Ingredients Journal of Japan. . 2010;215:53-58.

13. Bittner $\mathrm{S}$. When quinones meet amino acids: chemical, physical and biological consequences. Amino Acids. 2006;30(3):205-224.

14. Birben E, Sahiner UM, Sackesen C, Erzurum S, Kalayci O. Oxidative stress and antioxidant defense. World Allergy Organ J. 2012;5(1):9-19.

15. Bendary E, Francis RR, Ali HMG, Sarwat MI, El Hady S. Antioxidant and structure-activity relationships (SARS) of some phenolic and anilines compounds. Annals of Agricultural Sciences. 2013;58(2):173-181.

16. Hamaguchi T, Ono K, Murase A, Yamada M. Phenolic compounds prevent Alzheimer's pathology through different effects on the amyloid-beta aggregation pathway. Am J Pathol. 2009; 175(6):2557-2565.

17. Mirhashemi SM, Aarabi M-H. Effect of two herbal polyphenol compounds on human amylin formation and destabilization. $J$ Med Plant Res. 2012;6(16):3207-3212. 